\title{
Changes in social communication and mental health status among university students during the COVID-19 pandemic in 2020 in Japan
}

Momoko Nagai-Tanima ( $\nabla$ tanima.momoko.8s@kyoto-u.ac.jp )

Kyoto University

Yu Odake

Kyoto University

Kanako Shimoura

Kyoto University

Tomoki Aoyama

Kyoto University

\section{Research Article}

Keywords: COVID-19, social communication, communication methods, mental health, depression, anxiety, loneliness, university students

Posted Date: January 25th, 2022

DOI: https://doi.org/10.21203/rs.3.rs-1193857/v1

License: (9) This work is licensed under a Creative Commons Attribution 4.0 International License.

Read Full License 


\section{Abstract \\ Background}

The pandemic due to the coronavirus disease 2019 (COVID-19) has brought the change of social communication. For university students, friendships considered to be important to reduce stress and mental difficulties, and a decrease in social interaction with friends may have an impact on their mental health. This study aimed to investigate the association between changes in social communication during the COVID-19 pandemic and mental health status among university students in Japan.

\section{Methods}

This study followed a cross-sectional observational design using an online questionnaire survey. The participants were undergraduate and graduate students in Japan. The questionnaire was distributed only once between October 12 and 31,2020. Of the 194 respondents who agreed to participate in the study, 149 who completed their online survey were included in the analysis.The Hospital Anxiety and Depression Scale and the Japanese version of the Three-item Loneliness Scale were used to assess depression, anxiety, and loneliness. Regarding social communication, participants asked the frequency of contact with friends and family by methods (in-person, phone call, and text) and the number of people who have been communicated with. Responses were also obtained for communication during the same period in the previous year to determine changes between the two periods. A multiple regression analysis was conducted for depression, anxiety, and loneliness scores as dependent variables.

\section{Results}

The ratios of positive for depression and anxiety were $38.3 \%$ and $34.2 \%$, respectively. For communication with friends, changes in frequency were negatively associated with loneliness, and changes in number were negatively associated with depression and loneliness. Regarding text communication, a change in number was negatively associated with loneliness. For communication with families, a change in the number of people regarding text communication was positively associated with anxiety.

\section{Conclusion}

The impact of students' communication with friends and families on their mental health may need to be considered before conducting university initiatives to prevent COVID-19.

\section{Introduction}

Since the first coronavirus disease 2019 (COVID-19) case was confirmed in Wuhan, China, toward the end of $2019,[1]$ more than 70 million people had been infected and more than 1.6 million had died worldwide 
as of December 15, 2020.[2] The stay-at-home order was enforced in many countries to minimize the risk of infection by ensuring physical distance between people, refraining from going out to densely crowded places, and teleworking from home whenever possible. In Japan, the state of emergency, including social distancing, was implemented from April 7 to May 25, 2020, to curb the spread of new infections.[3] Policies to prevent the spread of COVID-19 have caused a wave of change in personal lives. Even trivial activities such as having a face-to-face conversation with friends, going out to dinner or traveling with family, or going to a public place such as a sporting event or an artist's concert are being altered to promote social distancing.[4]

Our connection with others has a significant impact on our lives. Previous research has shown that communication with family and friends and participation in social communities can improve quality of life and reduce the risk of developing mental health problems such as depression.[5, 6] With regard to communication methods, previous studies conducted in 2015 have shown that in-person communication contributes to a decreased risk of developing depression, while media such as phone calls and texting are not effective in preventing the risk of developing depression.[6] In addition, several reports have suggested that communication using digital devices may not be a substitute for face-to-face communication, which may lead to a decrease in communication ability and quality. $[5,7,8]$ Under the limited in-person communication circumstances during the pandemic, many reports have shown that the current situation is adversely affecting global human connection and mental health, including loneliness, stress, and anxiety, for people worldwide.[9-13]

The prevalence of COVID-19 has also brought significant changes in terms of communication with university students worldwide.[11, 13, 14] In July 2020, many universities in Japan were offering online and/or long-distance learning. In October 2020, after removing the state of emergency, approximately $19.3 \%$ of all universities offered face-to-face classes.[15] In addition, some universities were still restricting travel, going back to their hometown, and extracurricular activities to minimize the risk of infection.

In general, university students may likely face psychosocial stress and mental health problems because their generation is perceived as one that leaves their hometown to start an independent life[16] and is in a transitional period of social life, such as forming a new network of friends.[17] Friendships are also considered to be important for university students to reduce stress and mental difficulties,[18] and a decrease in social interaction with friends may have an impact on their mental health. Moreover, several studies have shown that the current COVID-19 situation has caused poor mental health. Tahara reported that isolation and loneliness of students, particularly for those living alone, could be the major reason for the decline in mental health.[19] Another report showed that exercise and having someone to consult about worries were associated with a decreased risk of depression symptoms and suicidal ideation.[12] No study has considered the relationship between mental health and communication details, such as the people and methods involved, in this age range. 
The purpose of this study was to investigate the relationship between changes in communication that focus on the object person, methods of communication, communication with others, and mental health status among university students. We hypothesized that changes in in-person communication with both parents and friends are more negatively associated with mental health risk than in text communication.

\section{Materials And Methods}

Study design

This was a cross-sectional observational study that used an online survey. The questionnaire contained items about present (current year) and past (previous year) scenarios. The degree of change was calculated by comparing the values of the differences.

\section{Setting}

Data acquisition and analysis were performed at the Kyoto University (Kyoto, Japan). The questionnaire was distributed once between October 12 and 31, 2020, and responses were collected during this period. The link of the online survey was distributed among students at Kyoto University through a chat application (e.g., LINE) with the help of the university's health care department. This study was approved by the Medical Ethics Committee of Kyoto University (approval no.: R2633).

\section{Participants}

The participants in this study were undergraduate and graduate students in the Kyoto University; only those who were at least 18 years old and not on a leave of absence were eligible for participation. We estimated the number of participants before the distribution of the questionnaire as follows: The null hypothesis was that the decreased frequency in in-person communication with family and friends during the COVID-19 pandemic is not associated with a high risk of depression or anxiety disorder, as compared to an increased or unchanged frequency, and the alternative hypothesis was two-sided. The required sample size was calculated with a minimum correlation coefficient $r$ of 0.30 , two-sided probability of alpha error of $5 \%$, and probability of beta error of $10 \%$. Assuming a withdrawal rate of approximately $25 \%$ for the online questionnaire, the required sample size was 151.

\section{Measurements}

The online questionnaires were distributed and collected using a web-based platform called SurveyMonkey, which uses secure socket layer encryption to transmit survey responses from the respondent's computer to the server. Therefore, an individual could not be identified, and it prevented third parties from viewing or tampering the data. Each participant was allowed to submit only one completed questionnaire. The time required to complete the questionnaire was approximately $7 \mathrm{~min}$. The questionnaire consists of the following three sections. 
Data on age, gender, height, weight, course, faculties, living status, and marital status were extracted from the questionnaires. Body mass index (BMI) was calculated by dividing the weight in kilograms by the squared height in meters.

Communication with friends and families

The participants were asked to rate the frequency and number of people with whom they communicated (friends and family) by each method (in-person, phone, and text), with reference to methods used in a previous study.[6] In this study, videophones were included as part of "phone." Email and chat using social networking services (e.g., LINE, Instagram, Facebook, and Twitter) were included as part of "text." Family members were defined as those within the second degree of kinship, and friends included unmarried partners. Participants answered the same sets of questions about communication on two time points: this year and last year.

The question regarding frequency was phrased as, "Looking back on the last few months, how often did you do the following things <communication methods> with your friends or family on average?" The answers were scored on a 7-point scale (score = answer: $1=<1$ day/six months, $2=1-2$ days/six months, 3 =1-2 days/three months, 4 =1-2 days/one month, $5=1-2$ days/week, $6=3-5$ days/week, 7 = $\geq 6$ days/week). A higher score indicates a higher frequency in communication.

The question regarding the number of people communicated with was phrased as, "In the last month, how many friends or family members did you do the following things <communication methods> with?" The answers were scored on a 6 -point scale (score = answer: $1=0$ person $/ \mathrm{month}, 2=1$ person $/ \mathrm{month}, 3$ = 2 persons $/$ month, $4=3-4$ persons $/$ month, $5=5-8$ persons $/$ month, $6=\geq 9$ persons $/$ month). A higher score indicates communication with many people.

For each item, the amount of change in communication was calculated by subtracting the current score from the previous year's score.

Mental disorders and loneliness

To confirm the present mental health status (current year), two types of scales were used. Symptoms of depression and anxiety were investigated using the Japanese version of the Hospital Anxiety and Depression Scale (HADS), a self-administered questionnaire with 14 questions, seven of which were used to assess depression, and the rest were used to assess anxiety.[20] For each question, there were four response options, each scored from 0 to 3 points. Depression or anxiety was judged to be positive at a total score of 8 or more in each category.[21]

The Japanese version of the Three-item Loneliness Scale (TILS) was used to measure loneliness.[22] This scale consisted of three questions. Each question had three answer choices, and each answer was scored from 1 to 3 points (1 =low, 3=high). Higher total scores indicated high loneliness. 
To confirm the mental health status before the COVID-19 outbreak, self-reported mental health condition and past history were asked: "Have you had depression or any other mental illness before the COVID-19 outbreak (before December 2019)?"

\section{Statistical analytics}

For descriptive data, variables were presented as mean \pm standard deviation (SD) for continuous variables and percentages for nominal or ordinal variables.

A multiple regression analysis was conducted for depression, anxiety, and loneliness scores as dependent variables. The independent variable is the amount of change in each communication measure. Adjustment variables included gender, BMI, living status (living alone or not), and course (undergraduate or graduate students). These adjustment variables were selected because they have been associated with mental disorders in previous studies.[13, 23-25] Note that this analysis was conducted separately for each communication measure. As supplemental data, the correlation coefficient among each mental health measure is shown in Supplemental Table 1. To confirm the relationship between each adjustment variable and mental measurement, univariate analysis using the Mann-Whitney $U$ test between each mental measurement and gender, course, or living status was conducted (Supplemental Table 2). Data analyses were performed using JMP Pro 14.0 (SAS Institute, Cary, NC, USA). Statistical significance was set at $p<0.05$.

\section{Patient and public involvement}

Patients and the public were not involved in this study.

\section{Results}

Table 1 presents demographic and descriptive data. Of the 194 participants who agreed to participate in the study, 149 who completed the questionnaire were included in the analysis. The mean age was $22.4 \pm$ 2.68 , and $51.7 \%$ of the participants were women. Over half of the participants were undergraduates (58.4\%). Of the participants, $68.5 \%$ lived alone, and most participants were single. The ratios of positive for depression and anxiety were $38.3 \%$ and $34.2 \%$, respectively.

A significant correlation was confirmed between each mental health score at the 2020 time point during the COVID-19 pandemic (Supplemental Table 1). There was no significant difference between gender, living status, and mental health scores. Compared with undergraduate students, graduate students had significantly higher depression and anxiety scores $(p=0.03$ and $p=0.02$, respectively; Supplemental Table 2).

\section{Changes in communication before and during the COVID-19 pandemic}

Figure 1 shows the changes in communication with friends. Regarding in-person communication, fewer than half of the participants communicated more than six days a week during the pandemic period than 
during the pre-pandemic period. There was also a decrease in the number of those who performed inperson communication, with nine or more people in a month. For phone call communication, there was an increase in the number of people communicating more than once a month during the pandemic period than during the pre-pandemic period. In addition, the number of people communicating by phone calls with five to eight people per month has increased by approximately 10 times. For text communication, the number of people communicating with more than nine people per month increased by approximately 30 people during the pandemic period than during the pre-pandemic period.

Figure 2 shows the changes in communication with family members. There were moderate differences between the scenarios before and during the COVID-19 pandemic; however, this change was not as significant as that in the case of friends.

\section{Relationship between changes in communication and mental health status}

Table 2 shows the relationship between changes in communication, frequency or number with friends, and mental health scores using multiple regression analysis. For in-person communication, change in frequency was negatively associated with loneliness score $(\beta=-0.23, \mathrm{Cl}=-0.42$ to $-0.08, \mathrm{p}<0.01)$, and changes in frequency were negatively associated with depression $(\beta=-0.20, \mathrm{Cl}=-1.10$ to $-0.10, \mathrm{p}=0.02)$ and loneliness scores $(\beta=-0.27, \mathrm{Cl}=-0.57,-0.15, p<0.01)$. For phone call communication, changes in frequency and number were not associated with mental health scores. For text communication, a change in number was negatively associated with loneliness score $(\beta=-0.20, \mathrm{Cl}=-0.60,-0.08, p=0.01)$.

Table 3 shows the relationship between changes in communication, frequency or number with families, and mental health scores using multiple regression analysis. In-person and phone call communication showed no association with mental health scores. Furthermore, a change in the number of people regarding text communication was positively associated with anxiety scores $(\beta=0.18, \mathrm{Cl}=0.07$ to $1.89, \mathrm{p}$ $=0.04)$.

\section{Discussion}

This study examined the relationship between mental health and communication changes, frequency, and number during the COVID-19 pandemic in 2020, considering communication methods in university students. To the best of our knowledge, this is the first study to focus on changes in the number of people and the frequency of social communication during the COVID-19 pandemic, and it includes more recent popular forms of communication (video calls and social networking services) and mental health status among university students. The most important finding is that a decrease in in-person and text communication with friends is associated with mental health problems. Moreover, increased communication with family members was associated with anxiety.

First, we consider the estimates of mental health risks in the study population compared to previous studies conducted in the same age group before the COVID-19 pandemic. Compared to previous studies, 
the current sample population was more likely to have higher anxiety scores on the HADS, although depression and loneliness scores on the TILS were not substantially different.[20, 26]

For Japanese university students, a previous study showed that living alone could be the major reason for the decline in mental health during the COVID-19 pandemic.[19] However, there were no significant differences between the living status and mental health measurements in the current study. A previous study used the 12-item General Health Questionnaire (GHQ-12) to capture general mental health conditions. The current study used two types of questionnaire, HADS and TILS, to capture three types of mental health problems: depression, anxiety, and loneliness. Therefore, the mental health problems captured in each study were different. More details of the relationship between mental health conditions and communication were revealed in this study.

In this study, reduced communication with friends via text and in-person was associated with a higher risk of mental health problems. Previous studies showed that who individuals communicate with is associated with the risk of mental disorders, and it also depends on the age of the individual.[6] The results of this study support the assertion that communication with friends is particularly important to the mental health of university students. These students often belong to the generation that is known to leave their parents and start an independent life.[16] In addition, they often experience a transition period in their social life, as they form a new network of friends through university encounters.[17] Previous studies have shown that relationships with friends are an important factor in the mental health and social life of university students, and social support from friends may be one of the mechanisms that alleviate the difficulties and stresses associated with the transition period of social life.[18] Based on these facts, friendships are considered one of the most important assets in a transition period, such as life at the university, from a mental health perspective.[27]

Furthermore, increased communication with family members via text was associated with a higher risk of anxiety. Previous studies conducted during the COVID-19 pandemic suggest that increased family interaction time may have a positive impact on the mental health of college students.[28] Meanwhile, individuals with high social anxiety may actively engage more in online communication via the Internet as a compensatory behavior.[29] Because the present study was a cross-sectional study, the direction of causality cannot be predicted, but one possibility is that communication with family members increased as a compensatory behavior for anxiety. In the future, longitudinal studies should be conducted to clarify the causal relationship between anxiety and communication with family members.

In addition, the present study found that the impact on mental health depended on the method of communication. Previous studies conducted in 2015 have shown that in-person communication contributes to a decreased risk of developing depression, while media such as phone calls and texting are not effective in preventing the risk of developing depression.[6] It has also been suggested that communication using digital devices may not be a substitute for face-to-face communication, which may lead to a decrease in communication ability and quality. $[5,7,8]$ However, this study suggests that not only in-person communication but also text communication may have an impact on the mental health 
status of university students during a pandemic. This suggests that in cases where in-person communication is limited by social distance initiatives for pandemic prevention, communication via digital devices may have an effect similar to in-person communication. Furthermore, this may be influenced by the fact that, compared to previous studies, the current study was conducted among university students, who are more likely to use online communication tools such as smartphones.[30-32] In addition, recent developments in information and communication technology may be related to the comfort of videophone use (e.g., Zoom and Skype) and the ability to easily exchange texts with friends through social network services (e.g., LINE, Instagram, Facebook, and Twitter).

Currently, many universities worldwide are introducing new class systems, such as online classes.[14, 15, 33] While online classes can decrease the risk of infection through social distancing, they may cause psychological distress, decreased class motivation, and academic anxiety.[34, 35] In addition, the results of this study show that a decrease in the frequency of in-person communication can affect mental health in university students. One possible solution to this dilemma is to use classes as a catalyst to activate phone calls and text communication between students. In addition, face-to-face classes with infection control measures are useful from a mental health perspective.

\section{Strengths and limitations of the study}

The advantage of the present study is that it focuses on the details of communication. With recent developments in information and communication technology, this is the first study to focus on the relationship between mental health and communication methods during the pandemic.

Owing to the limitations of our study, consideration should be taken when interpreting the results. First, because of the study's cross-sectional nature, the direction of the relationship between social communication changes and mental health status remains unclear, and further longitudinal studies are required. Second, recall bias may have affected the validity of the information because respondents were asked to recall and respond to the last year's social communication. Finally, selection bias may have affected the generalizability of the results because the sample was recruited from a single university. In future studies, it is better to perform sampling using similar age groups, including other universities, and mental health status should be obtained at baseline as well.

\section{Conclusion}

This study examined the relationship between mental health and communication changes, including methods, frequency, and number, in university students during the COVID-19 pandemic in 2020. The findings of this study suggest that, in a sample of Japanese university students, changes in communication with friends (in-person and text) and families (text) during the COVID-19 pandemic were significantly associated with students' mental health status, such as depression, anxiety, and loneliness. The impact on students' communication with friends and families may need to be considered when addressing social initiatives in universities to prevent the spread of COVID-19. 


\section{Abbreviations}

COVID-19

coronavirus disease 2019

BMI

body mass index

HADS

the Hospital Anxiety and Depression Scale

TILS

the Three-item Loneliness Scale.

\section{Declarations}

\section{Ethics approval and consent to participate}

This study was conducted in accordance with the guidelines of the Declaration of Helsinki and approved by the Medical Ethics Committee of Kyoto University (approval number: R2633).

Informed consent was obtained from all participants in the study by reading the instructions provided at the beginning of the online questionnaire and checking the consent box. The research instruction explained the outline of this study, students' anonymity, and that the results of the responses would not interfere with any student's life.

\section{Consent for publication}

Not applicable

\section{Availability of data and materials}

The raw data supporting the conclusions of this study are available upon reasonable request from the authors.

\section{Competing interest}

The authors declare no conflict of interests.

\section{Funding}

This study was funded by an operating grant from Kyoto University (budget code: 021010).

\section{Author Contributions}

Y. O., K. S., and M. T. designed the study. Y. O. performed data collection. Y. O. and M.T. performed statistical analysis and wrote the manuscript. K. S., M. T., and T. A. contributed to the drafting and critical 
revision of the manuscript. T. A. founded and was responsible for the publication. All authors have read and approved the final version of the manuscript.

\section{Acknowledgments}

We would like to express our sincere thanks to all the participants who cooperated in the study, the students, the Health Department of Kyoto University for their help in distributing the questionnaires, and the laboratory members for their advice in submitting the study.

\section{References}

1. Huang $C$, Wang $Y, L i ~ X$, et al. Clinical features of patients infected with 2019 novel coronavirus in Wuhan, China. Lancet 2020;395:497-506. doi:10.1016/S0140-6736(20)30183-5.

2. World Health Organization. WHO coronavirus disease (COVID-19) dashboard I WHO coronavirus disease (COVID-19) dashboard. https://covid19.who.int/ (Accessed Dec 9, 2020) 2020b.

3. Ministry of Health Labor and Welfare. Government efforts against coronavirus infections. https://www.mhlw.go.jp/stf/covid-19/seifunotorikumi.html (Accessed Dec 9, 2021) 2021 a.

4. Ministry of Health Labor and Welfare. Practical examples of a "new way of life" assuming the coronavirus. https://www.mhlw.go.jp/stf/seisakunitsuite/bunya/0000121431_newlifestyle.html (Accessed Dec 9, 2021) 2021b.

5. Lee PSN, Leung L, Lo V, et al. Internet communication versus face-to-face interaction in quality of life. Soc Indic Res 2011;100:375-89. doi:10.1007/s11205-010-9618-3.

6. Teo AR, Choi H, Andrea SB, et al. Does mode of contact with different types of social relationships predict depression in older adults? Evidence from a nationally representative survey. J Am Geriatr Soc 2015;63:2014-22. doi:10.1111/jgs.13667.

7. Flaherty LM, Pearce KJ, Rubin RB. Internet and face-to-face communication: Not functional alternatives. Communication Quarterly 1998;46:250-68. doi:10.1080/01463379809370100.

8. Drago E. The effect of technology on face-to-face communication - JournalQuest 2015;6:2. http://www.inquiriesjournal.com/articles/1137/2/the-effect-of-technology-on-face-to-facecommunication (Accessed Dec 9, 2021).

9. Lebel $C$, MacKinnon A, Bagshawe $M$, et al. Elevated depression and anxiety symptoms among pregnant individuals during the COVID-19 pandemic. J Affect Disord 2020;277:5-13. doi:10.1016/j.jad.2020.07.126.

10. Zhang Z, Zhai A, Yang M, et al. Prevalence of depression and anxiety symptoms of high school students in Shandong Province during the COVID-19 epidemic. Front Psychiatry 2020;11:570096. doi:10.3389/fpsyt.2020.570096.

11. Villani L, Pastorino R, Molinari E, et al. Impact of the COVID-19 pandemic on psychological well-being of students in an Italian university: A web-based cross-sectional survey. Global Health 2021;17:39. doi:10.1186/s12992-021-00680-w. 
12. Nomura K, Minamizono S, Maeda E, et al. Cross-sectional survey of depressive symptoms and suicide-related ideation at a Japanese national university during the COVID-19 stay-home order. Environ Health Prev Med 2021;26:30. doi:10.1186/s12199-021-00953-1.

13. Cao W, Fang Z, Hou G, et al. The psychological impact of the COVID-19 epidemic on college students in China. Psychiatry Res 2020;287:112934. doi:10.1016/j.psychres.2020.112934.

14. Saha A, Dutta A, Sifat RI. The mental impact of digital divide due to COVID-19 pandemic induced emergency online learning at undergraduate level: Evidence from undergraduate students from Dhaka City. J Affect Disord 2021;294:170-9. doi:10.1016/j.jad.2021.07.045.

15. Ministry of Education Culture Sports Science and Technology. Implementation of classes at Universities on the Situation of Coronavirus Infection. https://www.mext.go.jp/a_menu/coronavirus/mext_00007.html (Accessed Oct 30, 2021) 2021.

16. Japan Student Services Organization. Students living survey in 2018. https://www.jasso.go.jp/about/statistics/gakusei_chosa/2018.html (Accessed Dec 9, 2021) 2018.

17. Buote VM, Pancer SM, Pratt MW et al. The Importance of Friends: Friendship and Adjustment Among 1st-Year University Students. Journal of Adolescent Research 2007;22:665-89.

18. Tokuno K. The early adult transition and friendships: Mechanisms of support - PubMed. Adolescence 1986;83:593-606. https://pubmed.ncbi.nlm.nih.gov/3812066/ (Accessed Dec 9, 2021).

19. Tahara M, Mashizume $Y$, Takahashi $K$. Mental health crisis and stress coping among healthcare college students momentarily displaced from their campus community because of covid-19 restrictions in japan. Int J Environ Res Public Health 2021;18. doi:10.3390/ijerph18147245.

20. Matthews-Ewald MR, Zullig KJ. Evaluating the performance of a short loneliness scale among college students. J Coll Stud Dev 2013;54:105-9. doi:10.1353/csd.2013.0003.

21. Olssøn I, Mykletun A, Dahl AA. The hospital anxiety and depression rating scale: A cross-sectional study of psychometrics and case finding abilities in general practice. BMC Psychiatry 2005;5:46. doi:10.1186/1471-244X-5-46.

22. Igarashi T. Development of the Japanese version of the three-item loneliness scale. BMC Psychol 2019;7:20. doi:10.1186/s40359-019-0285-0.

23. Seeman MV. Psychopathology in women and men: Focus on female hormones. Am J Psychiatry 1997;154:1641-7. doi:10.1176/ajp.154.12.1641.

24. Hidese S, Asano S, Saito K, et al. Association of depression with body mass index classification, metabolic disease, and lifestyle: A web-based survey involving 11,876 Japanese people. J Psychiatr Res 2018;102:23-8. doi:10.1016/j.jpsychires.2018.02.009.

25. Wang ZH, Yang HL, Yang YQ, et al. Prevalence of anxiety and depression symptom, and the demands for psychological knowledge and interventions in college students during COVID-19 epidemic: A large cross-sectional study. J Affect Disord 2020;275:188-93. doi:10.1016/j.jad.2020.06.034.

26. Liu Q, Shono M, Kitamura T. Psychological well-being, depression, and anxiety in Japanese university students. Depress Anxiety 2009;26:E99-105. doi:10.1002/da.20455. 
27. Rybak A, Mcandrew FT. How do we decide whom our friends are? defining levels of friendship in Poland and the United States. J Soc Psycho/ 2006;146:147-63. doi:10.3200/SOCP.146.2.147-163.

28. Alam MK, Ali FB, Banik R et al. Assessing the mental health condition of home-confined university level students of Bangladesh due to the COVID-19 pandemic. Z Gesundh Wiss 2021:1-8.

29. Weidman AC, Fernandez KC, Levinson CA, et al. Compensatory internet use among individuals higher in social anxiety and its implications for well-being. Pers Individ Dif 2012;53:191-5. doi:10.1016/j.paid.2012.03.003.

30. Gross EF. Adolescent internet use: What we expect, what teens report. J Appl Dev Psychol 2004;25:633-49. doi:10.1016/j.appdev.2004.09.005.

31. Long J, Liu TQ, Liao YH, et al. Prevalence and correlates of problematic smartphone use in a large random sample of Chinese undergraduates. BMC Psychiatry 2016;16:408. doi:10.1186/s12888-0161083-3.

32. Parasuraman S, Sam AT, Yee SWK, et al. Smartphone usage and increased risk of mobile phone addiction: A concurrent study. Int J Pharm Investig 2017;7:125-31. doi:10.4103/jphi.JPHI_56_17.

33. Driessen E, Beatty A, Stokes A, et al. Learning principles of evolution during a crisis: An exploratory analysis of student barriers one week and one month into the COVID-19 pandemic. Ecol Evol 2020;10:12431-6. doi:10.1002/ece3.6741.

34. Al-Tammemi AB, Akour A, Alfalah L. Is it just about physical health? An online cross-sectional study exploring the psychological distress among university students in Jordan in the midst of COVID-19 pandemic. Front Psychol 2020;11:562213. doi:10.3389/fpsyg.2020.562213.

35. Horita R, Nishio A, Yamamoto M. The effect of remote learning on the mental health of first year university students in Japan. Psychiatry Res 2021;295:113561. doi:10.1016/j.psychres.2020.113561.

\section{Tables}

Tables 1-3 are available in the Supplementary Files section.

\section{Figures}


Frequency of in-person communication with friends

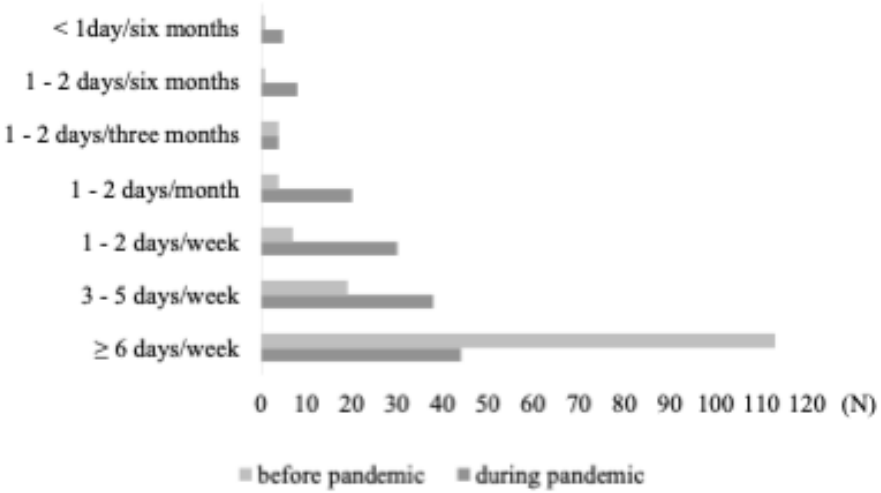

Frequency of phone communication with friends

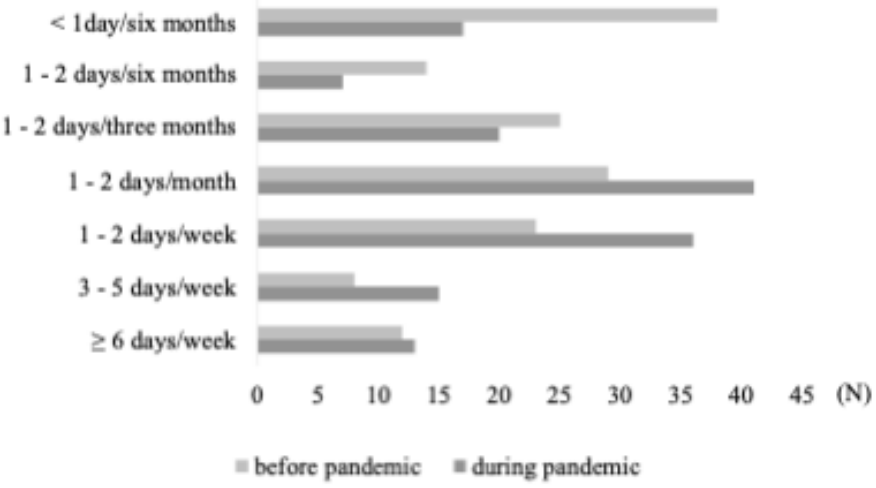

Frequency of text communication with friends

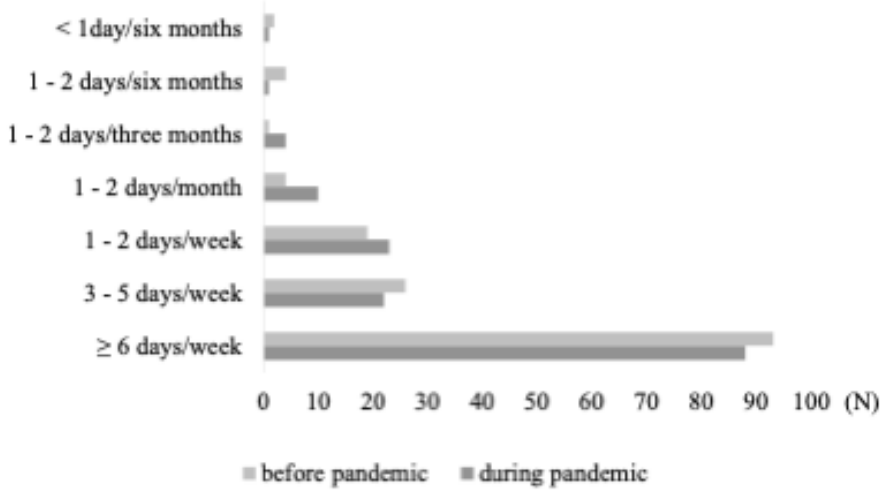

\section{Figure 1}

Number of friends with in-person communication

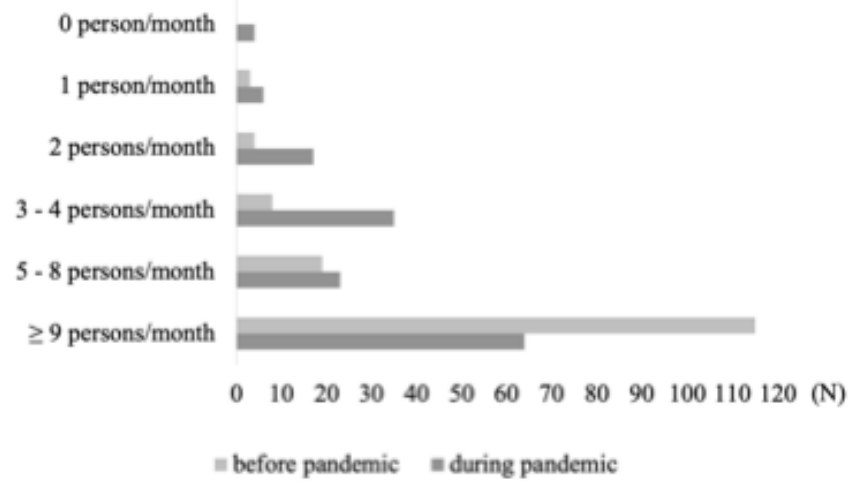

Number of friends with phone communication

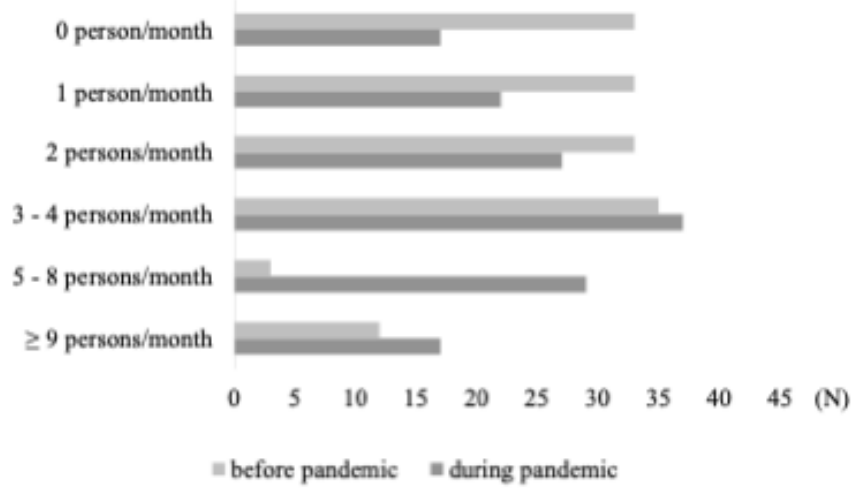

Number of friends with text communication

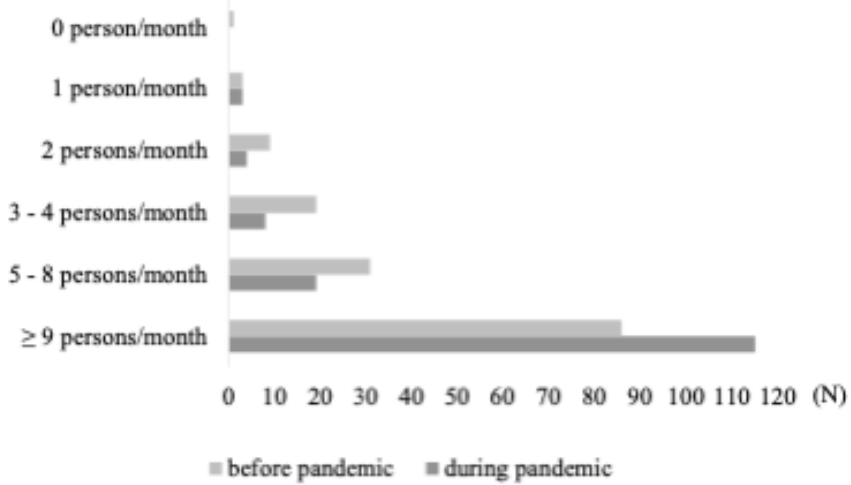

\section{Figure 1}

Changes in communication with friends

In-person communication, fewer than half of the participants communicated more than six days a week during the pandemic period than that of pre. For phone call communication, there was an increase in the number of people communicating more than once a month during the pandemic period than that of pre. 
In addition, that with five to eight people per month has increased by approximately 10 times. For text communication, the number of people communicating with more than nine people per month increased by approximately 30 people during the pandemic period than that of pre.

Frequency of in-person communication with families

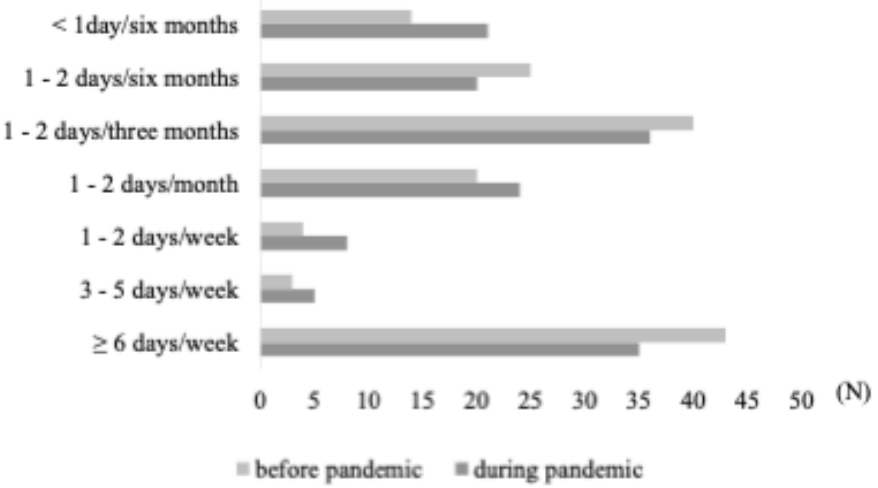

Frequency of phone communication with families

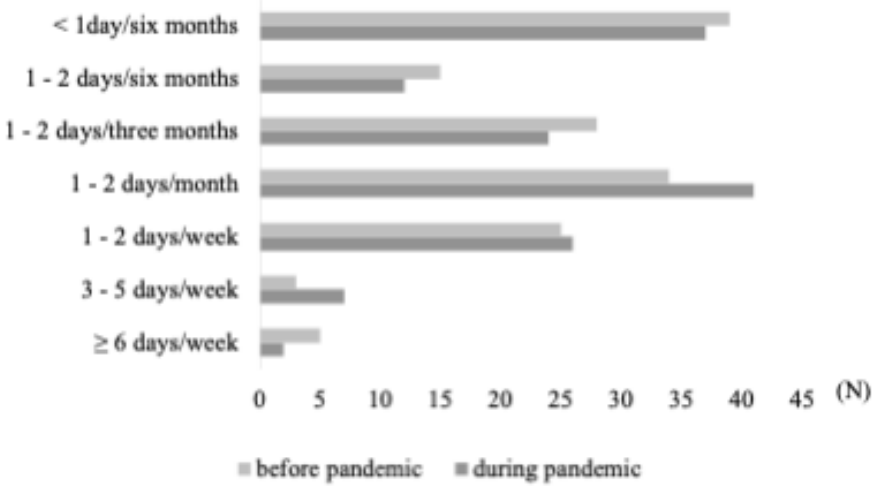

Frequency of text communication with families

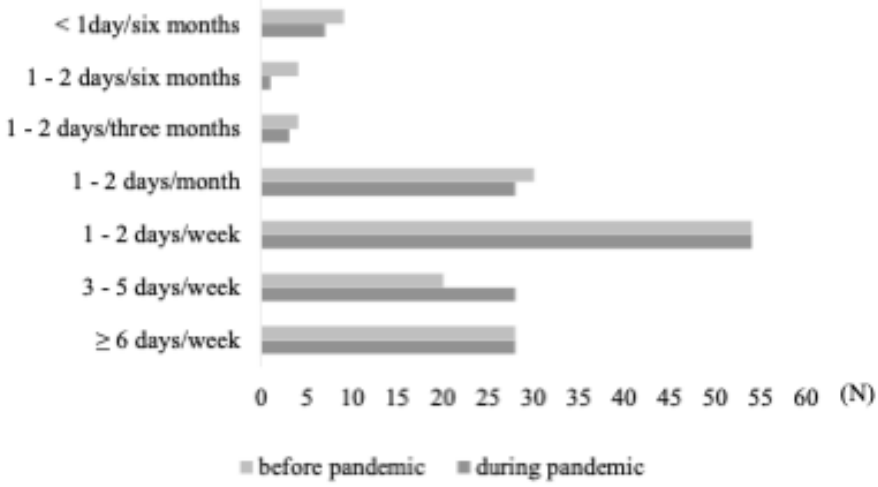

Figure 2
Number of families with in-person communication

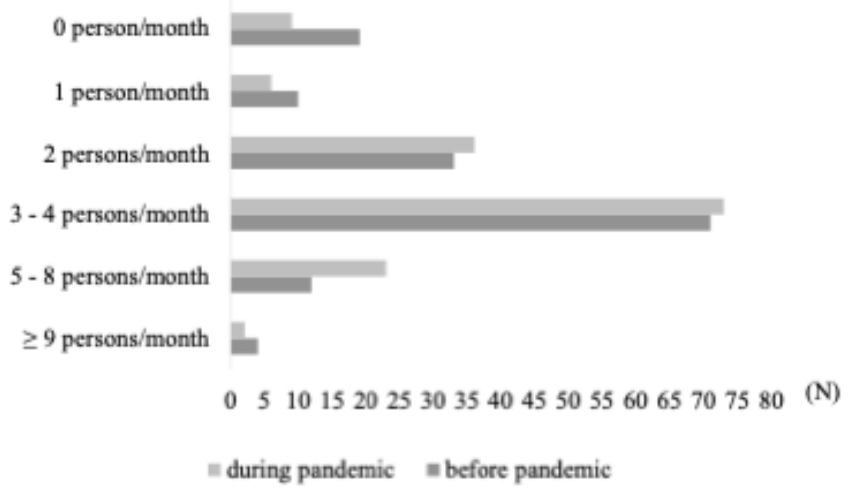

Number of families with phone communication

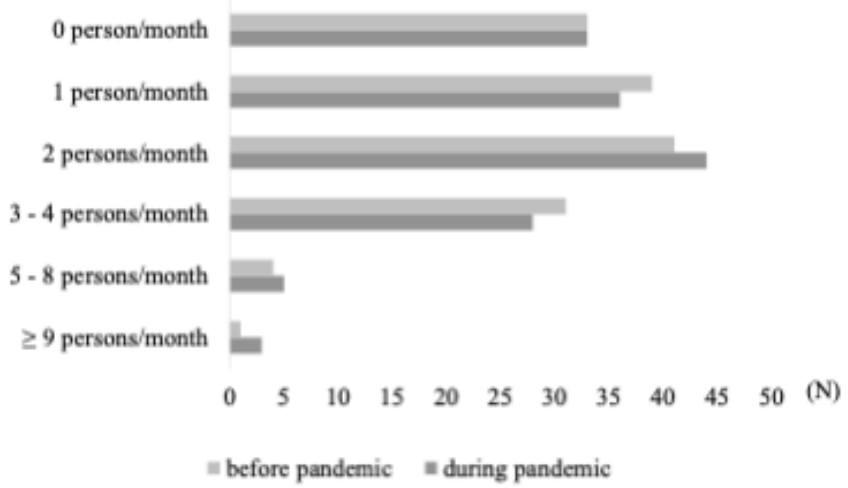

Number of families with text communication

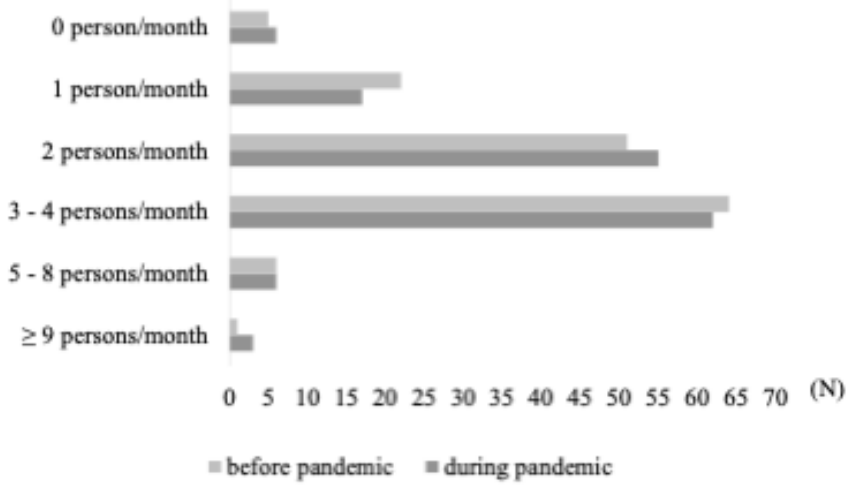

Figure 2

Changes in communication with family members. 
There were moderate differences between the scenarios before and during the COVID-19 pandemic; however, this change was not as significant as that in the case of friends.

\section{Supplementary Files}

This is a list of supplementary files associated with this preprint. Click to download.

- 211222Table1forsubmit.xlsx

- 211222Table2forsubmit.xlsx

- 211222Table3forsubmit.xlsx

- 211222SupTable1.xlsx

- 211222SupTable2.xlsx 